\title{
Managerial Skills for Managing Construction Safety
}

\author{
Sunindijo, R.Y. ${ }^{1}$, Zou, P.X.W. ${ }^{2}$, and Dainty, A.R.J. ${ }^{3}$
}

\begin{abstract}
Previous research has suggested that self-awareness, visioning, and sincerity are foundational managerial skills for delivering positive safety outcomes in construction projects. This paper aims to verify this finding and to suggest learning approaches for developing these skills in practice. Interviews with experienced construction practitioners were analysed thematically to find common themes. The thematic analysis confirms the necessity of the managerial skills identified in the previous research for managing construction safety and provides directions for construction organisations to improve safety learning. Existing approaches, which mainly adopt the cognitivism learning philosophy, may not be sufficient because the nature of learning in practice aligns with social constructivism, showing that learning occurs informally through interactions with people and artefacts at work instead of in a classroom-structured environment. Furthermore, although learning methods to develop safety skills have been suggested, there remains a need for better statistical evidence of the effectiveness of these methods in delivering safety outcomes.
\end{abstract}

Keywords: Construction safety management; learning process; managerial skills; safety research.

\section{Introduction}

The construction industry has a reputation as being one of the most dangerous industrial sectors, with accident and fatality rates being constantly much higher than the all-industry average. In Australia, the government encourages construction stakeholders to work together to address safety issues by implementing safety culture on worksites, and initiating a strategic framework for work health and safety training with clear priorities and evaluation [1]. There is still an urgent need to find new strategies and methods to further improve construction safety and achieve the goal of zero harm [2]. Although the construction industry has made improvements in safety through the application of systems, standards, and technology, more of the same will not further significantly improve safety performance [3], because, no matter how automated a production process or complex a management system is, people operate the process or the system. It is people who control production and sometimes must intervene when unplanned events occur. Statistics showed that up to $90 \%$ of all accidents are attributed to human error.

${ }^{1}$ Faculty of Built Environment, UNSW Sydney, NSW 2052, AUSTRALIA.

2 Faculty of Science, Engineering \& Technology, Swinburne University of Technology, John St, Hawthorn VIC 3122, AUSTRALIA.

${ }^{3}$ School of Civil and Building Engineering, Loughborough University, Loughborough, Leicestershire LE11 3TU, UNITED KINGDOM.

*Corresponding author; e-mail: r.sunindijo@unsw.edu.au

Note: Discussion is expected before November, $1^{\text {st }} 2017$, and will be published in the "Civil Engineering Dimension", volume 20, number 1, March 2018.

Received 04 July 2017; revised 08 August 2017; accepted 17 August 2017.
Simply put, people make mistakes and human error is an undesirable, but inevitable, aspect of everyday life. Furthermore, due to its labour-intensive and multi-stakeholder characteristic, human errors have greater impacts on safety performance of the construction industry [4]. Therefore, focusing on the human side of safety and improving the skills of those who are responsible for managing people in construction projects are practical ways for improving safety performance [2,5].

In construction organisations safety should not only be the responsibility of safety personnel and the top management team; every employee has to be involved and accountable for safety. At the project level, project management personnel (construction managers, project managers, site managers, site engineers, and site supervisors) have important roles to play. As the main conduit between top management and site workers, they need to be safety leaders and advocate the implementation of safety management system in their projects [6]. However, despite their important roles, Australian Chief Executive Officers (CEOs) believe that these key personnel are lacking a basic understanding of business operations and that there is a need for better skills in engaging and influencing the workforce in safety conversations [3]. The Cooperative Research Centre (CRC) for Construction Innovation also highlighted the need to identify the skills required for the future business environment and to develop and apply effective skill development programs for project management personnel [7]. Similarly, Master Builders Australia [8] stated that the responsibility for safe construction, maintenance, and repair should 
rest with those who have the direct ability to control and manage safety at the relevant time. Therefore, it is important to improve the skills of project management personnel to improve safety performance on sites. In essence, if organisations or individuals are to improve safety, it is crucial that project management team have the necessary skills to do so.

There are four managerial skills, comprising 16 components essential in construction project management [9]:

1. Conceptual skills (visioning; scoping; integration)

2. Human skills (emotional intelligence; interpersonal skill; transformational leadership)

3. Political skills (apparent sincerity; social astuteness; networking ability; interpersonal influence)

4. Technical skills (scheduling; budgeting; quality management; risk management; document and contract administration; procurement management)

These four skills were tested using structural equation modelling (SEM) to identify the most important skills for implementing safety management tasks and developing safety climate. The finding, as depicted in Figure 1, shows that self-awareness, visioning, and apparent sincerity are the foundational skills and are the precursors of the first-tier mediator skills consisting of scoping and integration, and self-management. The second-tier mediator skills are social awareness, social astuteness, and relationship management. These skills support the implementation of safety management tasks and the development of positive safety climate [2].

Building upon this study, this current research explores the relevance and applicability of the model from the perspective of industry practitioners. The important skill components and their roles on construction safety management are discussed from the existing literature and practitioners' perspectives. Furthermore, the research explores the learning process in practice and identify skill development methods that complement the process. Lastly, discussion on how higher education institutions (e.g. universities) and safety research can contribute to improve safety in the construction industry is also included.

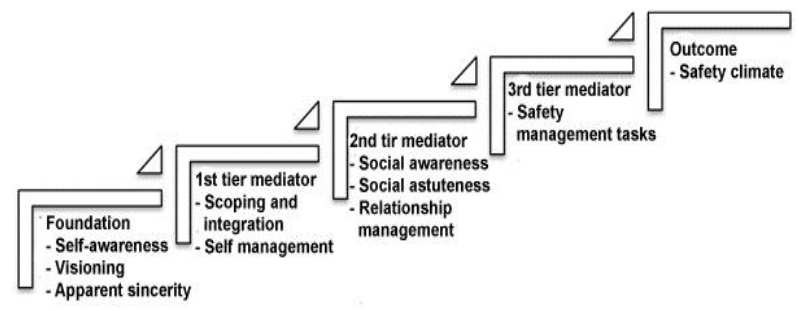

Figure 1. Managerial Skills for Managing Construction Safety

\section{Research Methods}

Qualitative research methodology, rooted in the interpretivist philosophical assumption, was adopted to confirm the model shown in Figure 1, gain richer understanding in interpreting the model, and obtain information for future research. Semi-structured interviews were used to collect qualitative data which were needed to obtain interpretations and perspectives held by experienced construction practitioners in the construction industry. It was expected that these interviews help to verify the research results, making them relevant to meet the current needs in the construction industry. The interview questions were as follows:

1. Based on previous survey, these are the most important skills for project management personnel to implement safety management tasks and develop safety climate (show the model). What is your opinion?

2. What is the most important skill for project management personnel to lead safety implementation?

3. What is the common issue that hinders safety performance improvement in the Australian construction industry?

4. In your opinion, is there a gap in safety implementation in your organisation? For example, management has certain expectations in the implementation of safety policies and safety management system, but the implementation at the project or site level may not meet these expectations.

5. What does your organisation do to improve the knowledge and skills of project management personnel so that to improve safety performance? (After the answer is given) Is the current safety training and induction effective?

6. How can universities and research contribute to improve construction safety?

7. Actually there has been a lot of research in construction safety conducted by universities, but the problem is that research results have not been embraced by the industry practitioners. The industry seems reluctant to implement the research results. Is this true and how can collaborations between universities and construction organisations be improved?

Purposive sampling was used to select the interview participants in this research. This is a non-random sample in which a range of methods was used to select cases of a specialised population [10]. A total of eight interviews were conducted and the profile of the participants is presented in Table 1. A thematic analysis of the data collected from the interview found only a few new codes from the last interview, 
which indicate theme saturation and that the number of interviews was adequate. Further, a small sample size may also be sufficient to find the general pattern of feelings or thoughts of individuals from similar environment of interest, which was the case in this research [11]. In addition, the interview sample represents various positions in construction projects. This variety of positions is advantageous for the research, because it enables the proposed relationships to be verified by industry practitioners from different management functions. The interview participants had an average of 21.8 years of experience in the construction industry, thus ensuring that they had sufficient experience of construction safety issues across different contexts. The researchers acknowledge that the sample size is small, thus the findings of this exploratory work must be cautiously interpreted and applied. As such, the findings were cross-referenced with the existing literature and theories to ensure their validity.

Table 1. Profiles of the interview participants

\begin{tabular}{|c|c|c|c|c|}
\hline Code & Position & Age & Education & $\begin{array}{c}\text { Experience } \\
\text { (years) }\end{array}$ \\
\hline CAM-01 & $\begin{array}{l}\text { Commercial } \\
\text { administration } \\
\text { manager }\end{array}$ & $50-54$ & Bachelor & 37 \\
\hline PM-02 & Project manager & $45-49$ & Bachelor & 24 \\
\hline HSEM-03 & $\begin{array}{l}\text { Branch EHS } \\
\text { manager }\end{array}$ & $45-49$ & Diploma & 28 \\
\hline PE-04 & Project engineer & $25-29$ & Bachelor & 5.5 \\
\hline CM-05 & $\begin{array}{l}\text { Construction } \\
\text { manager }\end{array}$ & $35-39$ & Bachelor & 19 \\
\hline CE-06 & $\begin{array}{l}\text { Commissioning } \\
\text { engineer }\end{array}$ & $>55$ & Diploma & 36 \\
\hline SM-07 & Site manager & $40-44$ & $\begin{array}{l}\text { High } \\
\text { school }\end{array}$ & 15 \\
\hline HSEM-08 & $\begin{array}{l}\text { National HSE } \\
\text { manager }\end{array}$ & $40-44$ & Diploma & 10 \\
\hline \multicolumn{4}{|c|}{ Average work experience $=$} & 21.8 \\
\hline
\end{tabular}

The interviews were conducted either face to face or by phone, and audio-recorded, transcribed, and analysed using the principles of thematic analysis. Thematic analysis is a process of encoding qualitative information [12]. It is a method for identifying, analysing, and reporting patterns or themes within data [13]. A theme is a pattern found in the data that at minimum describes and organises the possible observations and at maximum interprets aspects of the phenomenon. A theme may be identified at the manifest level, which is directly observable in the data, or at the latent level, which underlies the phenomenon. Thematic analysis is flexible and has a wide variety of uses, such as seeing a phenomenon from another person's perspective; making sense out of seemingly unrelated material; analysing qualitative information; systematically observing a person, an interaction, a group, a situation, an organisation, or a culture; and converting qualitative information into quantitative data [12].

The thematic analysis was performed based on the six stages proposed by Braun and Victoria [13]. The detail thematic analysis process performed is as follows:

1. The first stage was data familiarisation where all interviews were transcribed and reread for accuracy and data immersion. During the rereading process, notes and ideas were jotted down inductively.

2. In the second stage, initial codes from the data were generated and matched with the data extracts, which resulted in the identification of 51 codes.

3. Stage three involved sorting the codes into potential themes, which resulted in the identification of four themes. Relationships between themes and subthemes were also considered in this stage.

4. In stage four, the themes were reviewed and refined to ensure that data within themes cohere together meaningfully and that there are clear distinctions between themes. During the process, some themes were discarded, some collapsed into each other, whilst others were broken down into separate themes or subthemes. Two themes and nine subthemes were identified in this stage.

5. Stage five was defining and naming the themes by identifying the essence of each theme and determining what aspect of the data that each theme captures. The story that each theme tells has to fit into the broader overall story about the data in relation to the research objectives, which are to confirm the skill development model, discuss relationships among research variables, and find insights regarding strategies to develop the essential skills. The final thematic map of the data is presented in Figure 2. The first theme is the confirmation of the skill development model (Figure 1). The important skill components and their application or development process were also discussed from the perspectives of the interview participants. The second theme is safety learning, skill development, and research, which aims to seek insights on how to develop managerial skills to that improve safety performance in the construction industry.

6. The last stage of the thematic analysis was reporting to convince readers of the merit and validity of the analysis. This reporting does not only describe the data, but is embedded with analytic narratives and accompanied with arguments related to the research objectives. 


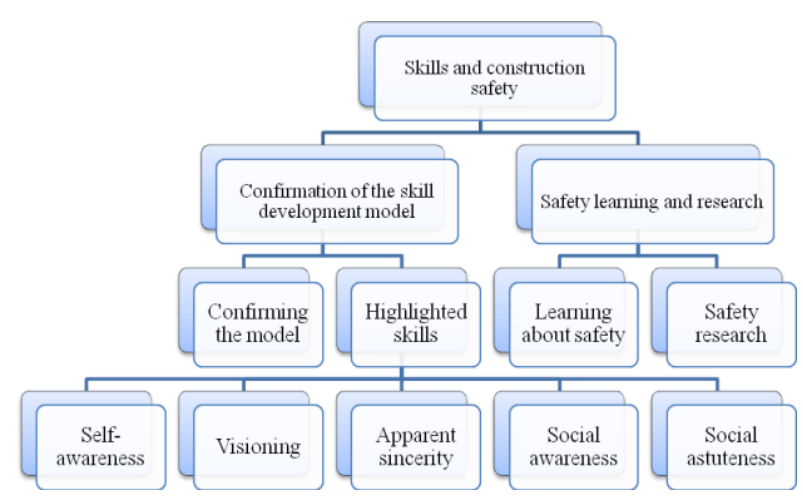

Figure 2. The Thematic Map of the Research

\section{Theme 1: Confirmation of the Skill Develop- ment Model}

\section{Interview Participants' Opinions Concerning the Model}

All interview participants supported the logic behind the final tiered skill development model as depicted in Figure 1. The model basically has answered the 'what' question by showing the skill components as well as the process to help individuals or construction organisations prioritise in developing the skill components. Two interview participants (HSEM-08 and CM-05) offered their opinion that this development is a process and it may not be prudent to skip tiers without being competent in the lower tier. For example, project management personnel should have strong foundational skills to stand on and build the higher-tier skill components. Underdeveloped foundational skills may undermine the effectiveness of other skill components, particularly during pressure and emergency situations where individuals tend to respond according to their most basic mindset.

The next question is that based on the competence level of an employee, which skill components have to be developed first. It is a question of evaluating the skills of every employee, thus the organisation can tailor a specific training and development program which meets the need of the employee.

The question of how to develop the skill components is more difficult to answer. Developing technical skill can be considered as straightforward, but developing 'soft' skills, such as emotional intelligence and political skill, is challenging. Existing literature has suggested approaches and methods to develop these 'soft' skills. Therefore, it is important to investigate systematically and objectively to prove the effectiveness of the approaches and methods. Conceptual skill, such as visioning, and scoping and integration, which can be influenced by various factors including experience, analytical ability, personal values, and other external factors, offers even bigger difficulties in its development process. Katz [14] suggested coaching, job rotation, and giving special assignments involving interdepartmental problems as methods to develop conceptual skill. However, their effectiveness may not have been sufficiently proven due to the limited attention given to conceptual skill, particularly in the construction industry. It appears that these methods may be superficial as they fundamentally only introduce certain tasks and ways to perform the tasks based on existing practices in the organisation (focus on behaviour). It does not consider, for example, the internal values and characters or personality of the person who undertakes the training.

\section{Skills Highlighted by Interview Participants}

Although the interview participants confirmed the validity of the model and the corresponding skills included in Figure 1, they were particularly drawn into five skills: self-awareness, visioning, apparent sincerity, social awareness, and social astuteness, and offered further clarification on their importance to manage construction safety. Self-awareness, visioning, and apparent sincerity were seen as the foundational skills that provide the necessary structure for the next tiers. The first foundation skill, self-awareness, is important because when people are aware of their values, strengths, and weaknesses, it will affect the way they manage themselves which will also be reflected on their actions. These actions, subsequently, will impact on the quality of relationships they build with others, which eventually determine their leadership effectiveness (interviewees HSEM-03 and CM-05). Previous studies also found that self-awareness is the core and starting point of self-management, relationship management, and effective leadership [15,16].

Visioning, as the second foundational skill, is essential because it is closely related to goal setting, both personal and organisational goals. In the context of this research, safety should be part of the overall vision in which "zero harm" is one of the key project objectives (interviewee PM-02). Maxwell [17] considered visioning or foresight as an indispensable quality of leadership. A vision is the energy that pushes through all difficulties. It unites and induces people to sacrifice for accomplishing goals. A maxim which says 'what you see is what you get' cannot be any truer in relation to this concept of visioning (interviewees HSEM-03, CM-05, and HSEM-08). Another important consideration about visioning is that the goals must be achievable and clearly expressed (interviewee CE-06). This is the basis to motivate and inspire people by providing meaning and challenge to their work [18]. Two interview 
participants (PE-04 and HSEM-08) emphasised the importance of clear goals and expectations in managing safety. It is important to realise that safety is a process, which will not happen overnight. Therefore, the goals and expectations should align with the capability of the human resource and the organisation in general. The vision needs to be grounded on where the organisation is and on its strength [18]. When a goal is beyond the capacity of the organisation, it would simply be nice words on a paper without any realisation in practice (interviewee $\mathrm{CE}-06$ ).

As leaders, project management personnel should not keep their vision to themselves, but also influence what is seen by others so that the vision also becomes what they are aspiring to achieve. In order to communicate this vision and make it a reality in the workplace, apparent sincerity, the third foundational skill, has an important role to play. When project management personnel put safety as one of the priorities and do not want anyone to get hurt, they have to say it and mean it. The vision should be communicated until the sincerity is felt by others (interviewees PM-02, HSEM-03, CM-05, and CE-06). Apparent sincerity is needed to communicate such vision effectively because project management personnel need to cultivate trust first before influencing people to accept the vision [17]. Eventually, the vision should become a governing principle or value in performing any work and activities, thus making safety one of the priorities in the project. This is the first step in creating safety culture that is embraced by everyone throughout the organisation. This result confirms the previous findings which suggested that leaders of complex organisations must have a strongly-held vision and communicate the vision to others so as to convert their vision into reality [18].

Three interview participants (CAM-01, PM-02, and SM-07) explained the importance of understanding people to lead safety implementation, which is about the social awareness and social astuteness skills in the context of this present research. The multistakeholder characteristic of construction projects and the diverse backgrounds of construction workforce make these skills crucial for project management personnel to communicate effectively, build trust, and embed safety values in others. One interview participant (CAM-01) gave a simple, but intriguing example on how a simple question 'do you understand?' can cause miscommunication, which may lead to a bigger problem. In responding to this question, when individuals from a certain culture say 'yes', it means 'yes, they understand'. On the other hand, for individuals from another culture, they may say 'yes' because of politeness, whilst in reality they actually do not understand or only understand partially. It is essential to remember that completing a project is about getting work done through others. Without the ability to understand people, it is difficult to relate to and get work done through them. Furthermore, the temporary nature of construction projects limits the luxury of gradually building long-term relationships with the team. Therefore, project management personnel must be able to quickly and accurately read and understand others to develop productive relationships for creating a safe workplace.

\section{Theme 2: Safety Learning and Research}

The interview participants explained how their organisations provide safety training for implementing standard safety measures to improve safety performance. Initially, everyone is trained to a minimum standard, for example, by using the Safety White Card Course, which has been mandated in Australia, requiring individuals to complete the course before they do any construction activities. Successfully completing this course is considered as a common denominator and an indication of basic safety competence in the Australian construction industry.

Achieving this minimum requirement is far from sufficient for construction organisations who aspire to uphold safety as one of their priorities. Consequently, construction organisations 'upgrade' their personnel's safety knowledge by authorising safety induction, which typically explains various hazards that may be encountered at work. There is also a tool box talk at the start of the day to make people aware of particular activities that will be performed in the project, along with the potential hazards, during that day. In addition, these organisations also have a supervision system to monitor and remind people to follow safety procedures.

All these training and learning methods are useful for developing safety knowledge. However, it is important to remember that safety learning and knowledge development is a process that does not happen instantaneously. There is a danger if construction organisations try to accelerate the rate of learning without realising it. Putting people in a room for five days to learn about safety and expect them to be experts, who would implement everything that they have learnt, is irrational. Therefore, it is important to determine the appropriate rate of learning to ensure its effectiveness by paying attention on different learning capacities of individuals (interview participants CM-05, CE-06, and HSEM08). 


\section{Learning-in-Practice}

Learning is clearly necessary for construction practitioners to perform their work safely. This learning is fundamental for maintaining and improving safety performance in the construction industry. Many organisations and training programs adopt the cognitivism approach to develop their human resource. Cognitivism examines how the mind makes sense out of stimuli in the environment and how information is processed, stored, and retrieved [19]. Cognitivism sees learning as being achieved by 'plucking an item from the tree of knowledge' [20]. Following this perspective, organisational learning literature and studies have focused on methods to codify, package and disseminate knowledge throughout organisations and workplaces. Many organisations also consider learning as an acquisition of knowledge which can be accomplished through classroom instruction and training. This view of learning considers that knowledge can be easily transferred from one medium to the minds of learners [21-23].

The interview participants questioned the effectiveness of the cognitivism approach in typical safety training. For example, CM-05 and CE-06 stated that it is unwise to depend on classroom training alone and expect people to know how to work safely. Although the cognitivism approach may seem logical and practical, critics argue that the resulting practices ignore the problems and complexities in the process of knowledge-making and learning. A countervailing learning theory is social constructivism which contends that learning is not a technological device aimed at capturing, processing, storing and disseminating data and information, but, rather, is situated in local practices where people collaborate and cooperate to solve daily issues [23]. Here, learning occurs through the observation of people in one's immediate environment and it is a function of the interactions between the person, the environment, and the behaviour [19]. In other words, learning involves taking part in the social world, i.e., learning takes place among and through others [22]. Learning should be seen not only as a product, but also as a process in which the learner is part of the environment [24]. Knowledge is integrated and distributed in every day's activities, thus learning cannot take place if participation in those activities is not possible [22].

The interview participants supported the importance of social constructivism perspective for promoting effective safety learning. CAM-01 and HSEM-08 highlighted the necessity of on-the-job informal training to educate workers on site. SM-07 explained the importance of mentoring in the field where project leaders are actually doing the talk and taking safety as part of their personal goals. It is also important to recognise that people have diverse ways of learning and that safety training should be flexible and tailored based on the learning needs of individual personnel (CM-05 and HSEM-08).

In the reality of construction projects, many problems do not neatly fit into predetermined categories, thus forcing construction practitioners to employ novel solutions and creative strategies to manage non-routine situations [25]. Saurin et al. [26] supported this view by stating that construction workers are often required to make important decisions in a dynamic work environment. This kind of work environment demands them to talk to each other about potential hazards while they are in action and employ their own adaptive accident prevention strategies without waiting for site management approval. Due to this reality, Wadick [25] implied that construction practitioners, especially workers, do not consider safety regulations, training, and research as something beneficial for them. They believe that many safety rules do not address their real safety concerns, but an attempt by powerful bureaucrats to subdue their subjects. As a result, they resist such instructions by doing as little as they can to comply, a far cry from the 'best practice' that those regulations, training, and research often try to achieve [25]. There is also an issue concerning the ineffectiveness of existing safety training programs. A study has shown that a classroom-like training setting only has short-term impacts on safety performance. After a short period of time, workers tend to forget what they have learnt and as a result safety performance returns to where it was before [27].

\section{Social Constructivism Approach to Safety Learning}

Rather than solely depending on 'arm's length' methods, there is a need to use approaches to safety learning that are more collaborative, and which prioritise dialogue and practical problem solving $[28,29]$. As stated previously, some interview participants (CAM-01, PE-04, CM-05, SM-07, and HSEM-08) aligned with this view and voiced the need to have a kind of on-the-job training and informal training/learning for safety. On-the-job training, in either formal or informal setting, is flexible and relevant to what people do in the organisation. It is a type of training in which an experienced employee adopts or mentors a new employee to teach him or her how to perform job duties [30]. It should be planned and follow a logical progression of stages, which includes preparing the trainees by putting them at ease, finding out what they know, and inciting their interests; presenting the information by telling, showing, and questioning 
one point at a time to ensure that the trainees understand; providing the trainees with practice by having them perform the tasks, observing and correcting, and evaluating; and lastly following up regularly before reducing supervision gradually as performance improves. In the case of on-the-job training, the supervisor or manager who conducts the training must be able to teach and show the trainees what to do [31].

On the other hand, informal training and learning occurs through interactions and feedback among employees as a result of a learning need in the context of working. Much of what people know about their jobs they learn informally from asking questions and getting advice from other employees and their supervisors, rather than from classroomstructured and formal training programs. Informal training may involve group problem solving, job shadowing, coaching, or mentoring [31]. As shown previously, a great deal of learning occurs informally, through interactions with people and artefacts at work, thus construction organisations should be able to harvest the advantages of informal learning by developing a work environment that promotes such learning. Construction organisations should also make sure that the correct contents are being used and exchanged during the informal learning process. Unsafe habits, shortcuts, and ways to bypass regulations, for examples, are not learning experience that organisations want to nurture. Therefore, the correct culture of safety has to be developed first and become the basis of both on-thejob and informal learning.

Previous studies in other disciplines have suggested methods to develop the important skills identified in this research, i.e., self-awareness, visioning, apparent sincerity, social awareness, and social astuteness. For example, it is suggested that keeping an 'emotion journal' to look for emotion patterns by recording events that trigger strong emotions along with the physical sensations that accompany the emotions is helpful to see oneself more objectively and improve self-awareness [16,32]. Action training is seen as a method to improve charismatic communication and apparent sincerity [33]. Social awareness can be improved by paying attention and actively looking for emotional clues from facial expression; the pitch, rhythm, and tone of voices; and the feelings conveyed by the posture of someone's body [34].

This research, therefore, offers another approach to learning about safety. The cognitivism approach currently dominates safety training in the construction industry. This approach is indeed useful and necessary, but is also inadequate to generate effective safety learning. Cognitivism should be complemented by social constructivism that promotes the view that safety learning occurs in practice through various informal ways. Social constructivism also recognises that every individual has a unique and preferred way of learning, indicating that safety training should be flexible and tailored based on the learning needs of individual personnel.

It should be pointed out that not much empirical evidence has shown the effectiveness of the abovementioned methods in practice, particularly in the context of construction safety. There is a need, therefore, to contextualise these methods, i.e., how, when, and where they should be employed, and determining their effectiveness. The longitudinal impacts of such training methods on various performance indicators, such as safety, employees' motivation, job satisfaction, and achievement of project or organisational objectives, can also be investigated. Furthermore, it is necessary to monitor how the newly developed skills are implemented in practice (if they are implemented in the first place). If there are more than one skill development methods, then future research can compare the effectiveness of these strategies and find out how they may complement, or conflict to, one another, particularly those that are currently being used in construction organisations.

It should be noted that "conventional research methods", i.e., questionnaire survey and interview, may not be adequate to collect the necessary data and validate the above mentioned future research. Observation and ethnography may be the more suitable methods to gain rich data which reflect what actually happens in practice. This aligns with the argument proposed earlier, showing that learning occurs in practice through interactions with people and artefacts at work. An interview participant (EHSM-03) addressed the importance of observing and finding out real issues on construction sites. Future safety research should be implemented in the form of longitudinal action-research to track a live project so that to develop grounded theories and provide recommendations at the completion of the research. Therefore, instead of explaining the cognitive process and conceptual structure involved in safety implementation, research should investigate what kind of social engagements provide a proper context for learning and improving safety (see for example [22]).

Finally, it is important to point out that the focus of learning and development is the person, i.e., the learner. Its aim is to enable an individual to develop his/her abilities and strengths to the fullest extent 
and to find individual achievement. The active participation, encouragement, and guidance from the superior and organisation are certainly needed for development efforts to be fully productive. However, it is important to remember that no one can motivate a person towards self-development if the person has no desire to develop. Motivation must come from within, i.e., intrinsic. Drucker [35] said that development is always self-development. For an organisation to assume responsibility for the development of a person/employee is idle boast. The responsibility rests with the individual, his/her abilities and efforts. From this motivation of selfdevelopment, a person will then realise the need to develop others. The best performers in any profession always look upon the people they have trained and developed as the proudest monument they can leave behind [35]. Similarly, in this present research, project management personnel must find motivation from within to develop themselves, otherwise no matter what methods, approaches, and training that the organisation uses will not be effective.

\section{Safety Research}

Universities have an important role in this learning process through their curriculums and research. Incorporating safety into these education programs is the first step to provide younger generations some basic principles on the importance and necessity of safety. This understanding enforces safety to be a part of their vision, a target that should be achieved, and hopefully will become a reality in their future projects. Education is crucial to implant safety values into people's mind-set as early as possible, which may lead to changes in behaviour (interview participants CAM-01, PM-02, and CM-05).

Another issue is a lack of collaboration between universities and the construction industry in conducting research. It appears that they reside in two different realms with little interactions. Research studies on construction safety normally start with the identification of a research gap through a literature review process. Once a gap is found, research objectives are formulated and the researchers are then 'let loose' to collect data that they need to analyse, discuss, and conclude. Although this may be sufficient from the academic perspective, but in practice only a few research results have been implemented because the results may not be relevant for, or meet the needs of, the construction industry.

This situation demonstrates that proper integration between theory and practice is required. It is imperative for universities to be proactive in building this collaboration (interviewees PM-02, HSEM-03, and
SM-07). They need to initiate communication by contacting and nurturing relationships with construction organisations. Not all organisations will be interested, but some will, and without being proactive, it is impossible for universities to identify these organisations that are willing to cooperate. Another aspect of research that should be considered carefully, which was raised repeatedly by the interview participants (HSEM-03, PE-04, CM-05, CE-06, and HSEM-08), is the practicality of research results. Researchers should consider observing what actually happens on sites to find problems and generate practical solutions for the participating organisations. It is possible, for example, that safety research is implemented in the form of longitudinal actionresearch to track a live for developing grounded theories and providing recommendations for improvement at the completion of the research. Such collaboration can be continued into the implementation or trial stage of the solutions to ensure proper integration of research results into the practices of the participating organisations.

Accordingly, an important focus will be on education and training providers to deliver relevant education programs for both fresh industry entrants and experienced practitioners [7]. From the research perspective, this shows the need to change the methodology and the philosophy in conducting research. This is the same perspective as has been discussed in the previous section arguing that learning occurs in practice and not merely by 'plucking information from the tree of knowledge'. Construction safety research should recognise the alternative paradigm where safety knowledge and learning are seen as social and cultural phenomena developed through interactions of individuals with each other and with non-human artefacts at work [25]. In other words, learning should not be seen merely as a product, but a process where the learner is part of the environment. This view of learning emphasises the context and the influence of cultural and social factors in the learning process [24]. Due to this reality of safety learning in practice, a modification to the methods of conducting construction safety research may be needed to investigate this alternative paradigm [22].

\section{Conclusion}

This paper has discussed four areas which can help improve safety performance in the construction projects. First, a quantitative study has identified a set of interrelated managerial skills (Figure 1) to implement safety tasks and develop safety climate. In this present research, the relevance of these skills has been confirmed by experienced construction practitioners. Discussions and examples have been provided to show the importance of these skills in practice. 
Second, it is important to realise that learning-inpractice is not as simple as transferring knowledge from a medium into the mind of the learners, but it happens through interactions with people and artefacts at work. As such, cognitivism learning approach which is currently widely adopted in practice, although is essential, is insufficient to develop construction workforce who will take lead in improving safety.

The learning-in-practice discussion leads to the third area concerning skill development methods. Construction organisations have to consider the process of learning-in-practice to ensure that their human resource development efforts are effective. They should realise that people learn much about their jobs informally from asking questions and getting advice from others, rather than from classroomstructured and formal training programs. Therefore, the culture of safety learning has to be promoted first to provide a proper learning environment. Furthermore, this study suggested different methods to develop the essential skills identified in this research. Future research should continue by contextualising their application and measuring their effectiveness.

Fourth, universities can contribute to the safety learning process through their curriculums and research. A modification to the methods of conducting construction safety research may be needed to integrate the realms of theory and practice to ensure that research findings are relevant and applied in practice.

\section{References}

1. ACT Government, Getting Home Safely: Inquiry into Compliance with Work Health and Safety Requirements in the ACT's Construction Industry, ACT Government, Canberra, 2012.

2. Zou, P.X.W. and Sunindijo, R.Y., Strategic Safety Management in Construction and Engineering, Wiley-Blackwell, Chichester, 2015.

3. Wagner, P., Safety - A Wicked Problem, 2010, Retrieved from http://www.peterwagner.com.au/ wp-content/uploads/Safety-A-Wicked-Problem2. pdf.

4. Lingard, H. and Rowlinson, S., Occupational Health and Safety in Construction Project Management, Spon Press, Oxon, 2005.

5. Reason, J., Human Error, Cambridge University Press, New York, 1990.

6. Dingsdag, D.P., Biggs, H.C., Sheahan, V.L., and Cipolla, D.J., A Construction Safety Competency Framework: Improving OH\&S Performance by Creating and Maintaining a Safety Culture, Cooperative Research Centre, Barton, 2006.
7. Hampson, K. and Brandon, P., Construction 2020: A Vision for Australia's Property and Construction Industry, Cooperative Research Centre for Construction Innovation, Brisbane, Australia, 2007.

8. Master Builders Australia, Building a Safer Future: Master Builders Occupational Health and Safety Policy Blueprint 2009-2015, Master Builders Australia, Yarralumla, 2010.

9. Sunindijo, R.Y., Project Manager skills for Improving Project Performance, International Journal of Business Performance Management, 16(1), 2015, pp. 67-83.

10. Neuman, W.L., Social Research Methods: Qualitative and Quantitative Approaches, 7th ed., Pearson, Boston, 2011.

11. Creswell, J.W., Qualitative Inquiry and Research Design: Choosing among Five Approaches, Sage Publications, Thousand Oaks, 2007.

12. Boyatzis, R.E., Transforming Qualitative Information: Thematic Analysis and Code Development, SAGE Publications, Thousand Oaks, 1998.

13. Braun, V. and Victoria, C., Using Thematic Analysis in Psychology, Qualitative Research in Psychology, 3(2), 2006, pp. 77-101.

14. Katz, R.L., Skills of an Effective Administrator, Harvard Business Review, Sep-Oct, 1974, pp. 90102.

15. Goleman, D., An EI-based Theory of Performance, In: Cherniss, C. and Goleman, D., Editors, The Emotionally Intelligent Workplace, Jossey-Bass, San Francisco, 2001, pp. 27-44.

16. Mersino, A.C., Emotional Intelligence for Project Managers: The People Skills You Need to Achieve Outstanding Results, AMACOM, New York, 2007.

17. Maxwell, J.C., Developing the Leader within You, Thomas Nelson, Nashville, 1993.

18. Hannagan, T., Mastering Strategic Management, Palgrave, Hampshire, 2002.

19. Merriam, S.B. and Caffarella, R.S., Learning in Adulthood: A Comprehensive Guide, JosseyBass, San Francisco, 1999.

20. Tsoukas, H. and Mylonopoulos, N., Introduction: Knowledge Construction and Creation in Organizations, British Journal of Management, 15(S1), 2004, pp. S1-S8.

21. Gherardi, S. and Nicolini, D., To Transfer is to Transform: The Circulation of Safety Knowledge, Organization, 7(2), 2000, pp. 329-348.

22. Gherardi, S. and Nicolini, D., Learning the Trade: A Culture of Safety in Practice, Organization, 9(2), 2002, pp. 191-223.

23. Styhre, A., Peer Learning in Construction Work: Virtuality and Time in Workplace Learning, Journal of Workplace Learning, 18(2), 2006, pp. 93-105. 
24. Hager, P., Conceptions of Learning and Understanding Learning at Work, Studies in Continuing Education, 26(1), 2004, pp. 3-17.

25. Wadick, P., Learning Safety in the Building Industry, 2006, Retrieved from http://www. cfmeu-construction-nsw.com.au/pdf/pwreslearnsafetybldgind.pdf.

26. Saurin, T.A., Formoso, C.T., and Cambraia, F.B., An Analysis of Construction Safety Best Practices from a Cognitive Systems Engineering Perspective, Safety Science, 46(8), 2008, pp. 1169-1183.

27. Laukkanen, T., Construction Work and Education: Occupational Health and Safety Reviewed, Construction Management and Economics, 17(1), 1999, pp. 53-62.

28. Bluff, L., Something to Think About-Motivations, Attitudes, Perceptions and Skills in Work Health and Safety, Safe Work Australia, Canberra, 2011.

29. Burke, M.J., Scheuer, M.L., and Meredith, R.J., A Dialogical Approach to Skill Development:
The Case of Safety Skills, Human Resource Management Review, 17(2), 2007, pp. 235-250.

30. Samson, D. and Daft, R.L., Management, $3^{\text {rd }}$ Asia Pacific ed., Cengage Learning, South Melbourne, 2009.

31. Mathis, R.L. and Jackson, J.H., Human Resource Management, South-Western Cengage Learning, Mason, 2011.

32. Bradberry, T. and Greaves, J., Emotional Intelligence 2.0, TalentSmart, San Diego, 2009.

33. Frese, M., Beimel, S., and Schoenborn, S., Action Training for Charismatic Leadership: Two Evaluations of Studies of a Commercial Training Module on Inspirational Communication of a Vision, Personnel Psychology, 56(3), 2003, pp. 671-697.

34. Caruso, D.R. and Salovey, P., The Emotionally Intelligent Manager: How to Develop and Use the Four Key Emotional Skills of Leadership, JosseyBass, San Francisco, CA, 2004.

35. Drucker, P., Management, Revised ed., Harper Collins, Pymble, 2008. 